\title{
Stellungnahme medswiss.net zur Managed-Care-Vorlage 04.062
}

\author{
Der Schweizer Dachverband der Ärztenetze begrüsst das vom Parlament verabschie- \\ dete neue Gesetz. Die anstehenden Probleme im Gesundheitswesen lassen sich nur \\ lösen, wenn alle Beteiligten zusammenarbeiten und Verantwortung übernehmen. \\ Die Räte haben das erkannt und im neuen Gesetz die entsprechenden Anreize vor- \\ gesehen. Teamarbeit wird honoriert. Der neue Artikel ist ein massvoller Schritt in die \\ richtige Richtung.
}

Jörg Fritschia, Alexander von Weymarn-Schärli ${ }^{b}$

a Präsident Verband Schweizer Ärztenetze medswiss.net

b Vizepräsident Verband Schweizer Ärztenetze medswiss.net
Korrespondenz: Dr. med. Jörg Fritschi medswiss.net Grütlistrasse 36 CH-8002 Zürich Tel. 0442804405 Fax 0442804403 medswiss.net@hin.ch

\section{Hauptzweck des neuen Artikels}

Managed Care, neu Integrierte Versorgung genannt, beruht nun auf einem Leistungsvertrag paritätischer Partner, nämlich der Versicherer und der Ärztenetze (neu: integrierende Versorgungsnetze). Der Vertrag regelt die Zusammenarbeit, den Datenaustausch, die Qualitätssicherung und die Vergütung der Leistungen.

Damit wird der bisherige Artikel 41.4 ersetzt, der den Versicherern das Recht gibt, die Leistungserbringer auszuwählen «im Hinblick auf eine kostengünstigere Versorgung».

Diese veraltete Formulierung hat massgeblich dazu beigetragen, dass Managed Care als Billigversicherung verstanden wurde. Das soll sich ändern.

\section{Verantwortlichkeiten für die integrierenden Versorgungsnetze}

Zur integrierenden Versorgung gehört neu die Bereitschaft der Versorgungsnetze, «eine Form von BudgetMitverantwortung» zu übernehmen. Form und Inhalt der Budget-Mitverantwortung sind durch die Vertragspartner des MC-Vertrages verhandelbar.

Die «älteren» Ärztenetze in unserem Land blicken auf eine über 12-jährige Erfahrung mit der Budgetmitverantwortung zurück. Die Zusammenarbeit mit den Versicherern ist von gegenseitigem Respekt geprägt.

\section{Verantwortlichkeiten für die Versicherten, Patientinnen und Patienten}

Die Versicherten lassen sich bei gesundheitlichen Problemen immer zuerst von ihrer frei wählbaren Netzärztin bzw. ihrem frei wählbaren Netzarzt beraten. Damit werden Doppelspurigkeiten und z.B. auch die falsche Wahl eines Spezialarztes vermieden. Deshalb profitieren die Versicherten von einer tieferen
Prämie und im Krankheitsfall von einem tieferen Selbstbehalt. Mit dem reduzierten Selbstbehalt wird die Integrierte Versorgung insbesondere für chronisch Kranke attraktiv.

Heute sind bereits mehr als 60\% der Hausärztinnen und Hausärzte vernetzt, Tendenz zunehmend. Damit ist eine angemessene Auswahl gewährleistet.

\section{Verantwortlichkeiten für die Versicherer}

Das neue Gesetz schreibt vor, dass die Integrierte Versorgung von Netzen angeboten wird, die versicherungsunabhängig sind. Damit können die Versicherer keine eigenen Praxen für Integrierte Versorgung mehr führen.

Die Versicherer verlieren auch ihr Recht, die Leistungserbringer auszuwählen, da der Art. 41.4 entfällt.

\section{Das konventionelle Versicherungssystem besteht weiter}

Keine Ärztin, kein Arzt ist gezwungen, ins ManagedCare-System einzutreten. Dies gilt auch für die Versicherten. Wer den vollen, unkoordinierten Zugang ins Gesundheitswesen will, zahlt im Vergleich zu heute den bescheidenen Aufpreis von 300 Franken pro Jahr (heutiger maximaler Selbstbehalt 700 Franken, neuer maximaler Selbstbehalt 1000 Franken).

Der Schweizer Dachverband der Ärztenetze dankt den Parlamentarierinnen und Parlamentariern, allen voran den Kommissionsmitgliedern für ihr Engagement für diese einvernehmliche und ausgewogene Gesetzeslösung als Grundlage für die integriert, sprich bedarfsgerecht koordinierte Gesundheitsversorgung in unserem Land. Koordination beruht letztlich auf Kooperation. Deshalb ist Managed Care / Integrierte Versorgung nicht nur ein Produkt, sondern auch eine Haltung! 